Journal of Engineering and Applied Sciences 14 (5): 1580-1584, 2019

ISSN: 1816-949X

(C) Medwell Journals, 2019

\title{
The Efficacy of Amaranth Extract as an Ingredient in Multi-Functional Cosmetics
}

\author{
${ }^{1} \mathrm{Hye}-\mathrm{Jin} \mathrm{Kwon}$ and ${ }^{2}$ Hyerroon Jang \\ ${ }^{1}$ Department of Chemical Engineering, Soongsil University, 369 Sangdo-ro, Dongjak-gu, Seoul, \\ Republic of Korea \\ ${ }^{2}$ Central Research Institute of Dr. Jmeelab, JMPMIO Co. Ltd., 31 Gaepor 7, Gangnam-gu, \\ Seoul, Republic of Korea
}

\begin{abstract}
This study assessed the anti-oxidative and anti-aging efficacies of amaranth extract and measured its applicability as a natural ingredient for multi-functional cosmetics. For this, the leaves and flowers of Korean native amaranth were extracted with $80 \%$ ethanol and two samples were obtained. After performing DPPH and ABTS assays, both collagenase and elastase inhibitory activities were measured. The data were then statistically analyzed. The results found the following: In terms of the antioxidant capacity of the two substances, both DPPH and ABTS radical scavenging activities revealed similar results which were dose-dependent. In collagenase and elastase inhibitory activities, the results were better than other natural materials that have recently been studied such as Eleutherococcus senticosus and Paeonia suffruticosa. Therefore, it was confirmed that amaranth extract would be suitable as an anti-aging ingredient that can prevent skin aging from ultraviolet rays or free-radicals. The said results prove the possibility of amaranth extract as a natural ingredient for multi-functional cosmetics. With the recent heightened interest in inner beauty, the development of an ingredient for cosmetics using food would be more meaningful.
\end{abstract}

Key words: Amaranth, multi-functional cosmetics, anti-oxidative, anti-aging inner beauty, Eleutherococcus senticosus, Paeonia suffruticosa

\section{INTRODUCTION}

Both "antioxidants" and "anti-aging" have recently emerged as prominent keywords in various sectors. The anti-aging trends in society, regardless of age and gender, coincide with the industrial activities of each sector and have become more evident. When it comes to the cosmetics industry, companies have spurred the development of functional products for anti-wrinkle, whitening and anti-aging effects (Valko et al., 2007). According to the Global Industry Market Research Report, the market value of anti-aging products could reach U\$275.2 billion in 2020 (Kwon, 2013). The anti-aging product market is expected to expand globally into Asian and African regions beyond Europe and the US and market growth appears to be further accelerated in response to the global aging phenomenon (Chung et al., 2003).

The local cosmetics industry has also helped Korea grow into the fourth largest cosmetics exporting country in 2017, promoting active research in various cosmetic ingredients (Omaye et al., 1997). Also, as the scope of functional skin care products increased from 3-11 categories, screening and regulatory requirements for safety and effectiveness have been reinforced (Lee et al., 2001). Amaranth belongs to the Amaranthus spp. L. family and is used as an ingredient for a wide variety of foods due to its significant nutritional properties including protein, squalene and polyphenol (Kim and Ryoo, 2002). Amaranth seeds are high in protein and minerals including iron, calcium, zinc, magnesium and lysine. The leaves are known to have large amounts of protein and ascorbic acid as well as flavonoid and anthocyanin pigments (Choi, 2011). In the wake of controversial events in relation to a toxic humidifier disinfectant accident, cancer causing chemicals in sanitary pads and heavy metals in skin care products, consumer reluctance to accept chemical products is increasing. On the other hand, the demand for natural ingredients and beauty products is growing, thereby boosting the number of chemical-free consumers who advocate for naturally occurring compounds with no hazardous chemicals (Han et al., 2013). In line with this trend in consumer markets, the grading and labelling system has been introduced to control patent applications and the safety of functional ingredients (Park et al., 2014). The present study,

Corresponding Author: Hye-Jin Kwon, Department of Chemical Engineering, Soongsil University, 369 Sangdo-ro, Dongjak-gu, Seoul, Republic of Korea 
therefore, aimed to evaluate the antioxidant and anti-aging effects of extracts from amaranth flowers and leaves which are widely used for functional foods and to develop a natural functional ingredient for skin care products.

\section{MATERIALS AND METHODS}

Extracts were obtained from Amaranth Leaves (AL) and Flowers (AF) grown in Pyeongchang, Gangwon Province, Korea through a preparation process in which the collected samples were thoroughly cleaned, cut into small pieces, naturally dried and ground. The $30 \mathrm{~g}$ of the prepared sample were placed into each extraction flask connected to a vertical reflux condenser and $250 \mathrm{~mL}$ of $80 \%$ ethanol was added to carry out extraction at 1.23 bar for $15 \mathrm{~min}$ in an autoclave (SJ-220A100, Sejong Scientific Co. Ltd., Bucheon, Korea). Vacuum filtration of the extract was then performed. The filtered liquid was condensed using a vacuum rotary evaporator (R-114, Buchi Co., Flawil, Switzerland) and dried using a freeze dryer (Ilshin Co., Seoul, Korea). Powdered samples were refrigerated until ready to use in the experiment.

DPPH assay: The 2, 2-diphenyl-1-picrylhydrazyl (DPPH) assay proposed by Brand-Williams was used to assess antioxidant capacity (Starkey et al., 1997). Two kinds of extracts were prepared at concentrations of $125,250,500$ and $1000 \mathrm{mg} / \mathrm{mL}$ and the DPPH solution was prepared at concentrations of $1 \times 10^{-4} \mathrm{M}, 2.5 \times 10^{-4} \mathrm{M}, 5 \times 10^{-4} \mathrm{M}$ and $6.5 \times 10^{-4} \mathrm{M}$. First, $1 \mathrm{~mL}$ of the sample solution was added to $1 \mathrm{~mL}$ of the DPPH solution for each concentration and absorbance at $540 \mathrm{~nm}$ was measured at $10 \mathrm{sec}$ intervals for $5 \mathrm{~min}$ using a UV/VIS spectrophotometer. After $25 \mathrm{~min}$ of incubation in the dark and standing for $5 \mathrm{~min}$, absorbance values were measured. Three replicates were prepared for each sample.

ABTS assay: Each of the $\mathrm{AL}$ and $\mathrm{AF}$ solutions was diluted to obtain final concentrations of $1,10,100$ and $1,000 \mu \mathrm{g} / \mathrm{mL}$. The ABTS solution was prepared by mixing $7.4 \mathrm{mM}$ ABTS with $2.6 \mathrm{mM}$ potassium persulphat and left to stand for one day in the dark to generate ABTS radical cation $(\mathrm{ABTS}+$ ). Then, the reaction mixture was diluted to obtain an absorbance of 1.5 or less at $732 \mathrm{~nm}$. After mixing $150 \mu \mathrm{L}$ of the diluted ABTS+ solution with $5 \mu \mathrm{L}$ of each $\mathrm{AL}$ and $\mathrm{AF}$ sample and incubated at room temperature for $10 \mathrm{~min}$ and then absorbance was measured at $732 \mathrm{~nm}$. Distilled water was added to the control sample and its ABTS + radical scavenging activity was calculated.

Elastase inhibition activity: Each $\mathrm{AL}$ and $\mathrm{AF}$ sample was diluted to make final concentrations of $1,10,100$ and $1,000 \mu \mathrm{g} / \mathrm{mL}$. A test solution was then prepared by dissolving elastase and N-succinyl-(L-Ala) 3-p-nitroanilide in a $50 \mathrm{mM}$ Tris- $\mathrm{HCl}$ buffer $(\mathrm{pH} \mathrm{8.6)}$ at each concentration level. Then, $40 \mu \mathrm{L}$ of $2.5 \mathrm{U} / \mathrm{mL}$ elastase was added to $5 \mu \mathrm{L}$ of each $\mathrm{AL}$ and $\mathrm{AF}$ sample and $80 \mu \mathrm{L}$ of $0.5 \mathrm{mg} / \mathrm{mL} \mathrm{N}$-succinyl-(L-Ala) 3-p-nitroanilide was added to each mixture which was then incubated at $37^{\circ} \mathrm{C}$ for $30 \mathrm{~min}$. After reaction, absorbance was measured at $445 \mathrm{~nm}$.

Collagenase inhibition activity: Each $\mathrm{AL}$ and $\mathrm{AF}$ sample was diluted to make final concentrations of 1 , 10,100 and $1,000 \mu \mathrm{g} / \mathrm{mL}$. To prepare a test solution, 4-phenylazobenzyloxycarbonyl-Pro-Leu-Gly-Pro-D-Arg was dissolved in $0.1 \mathrm{M}$ Tris-HCl buffer $(\mathrm{pH} 7.5)$ containing $4 \mathrm{mM} \mathrm{CaCl}_{2}$. Then, $250 \mu \mathrm{L}$ of $0.3 \mathrm{mg} / \mathrm{mL}$ 4-phenylazobenzyloxycarbonyl-Pro-Leu-Gly-Pro-D-Arg was added to $100 \mu \mathrm{L}$ of each $\mathrm{AD}$ and $\mathrm{AF}$ sample. The reaction was then initiated by adding $150 \mu \mathrm{L}$ of $0.2 \mathrm{mg} / \mathrm{mL}$ collagenase for $20 \mathrm{~min}$ at room temperature. After $500 \mu \mathrm{L}$ of $6 \%$ citric acid and $1.5 \mathrm{~mL}$ of ethyl acetate were added, absorbance was measured at $320 \mathrm{~nm}$.

Statistical processing: Experiment results were analyzed using an unpaired Student's t-test and ANOVA in SPSS 18.0 and statistical significance was determined with cutoffs at $\mathrm{p}<0.05, \mathrm{p}<0.01$ and $\mathrm{p}<0.001$

\section{RESULTS AND DISCUSSION}

\section{Results of the experiment and considerations}

DPPH assay: Total antioxidant capacity of Leaf and Flower extracts of amaranth (AL and $\mathrm{AF}$ ) is presented in Fig. 1. The DPPH radical scavenging ability of the AL extract was $22.2 \pm 0.6,43.0 \pm 0.4,69.3 \pm 0.2$ and $79.5 \pm 0.2 \%$,

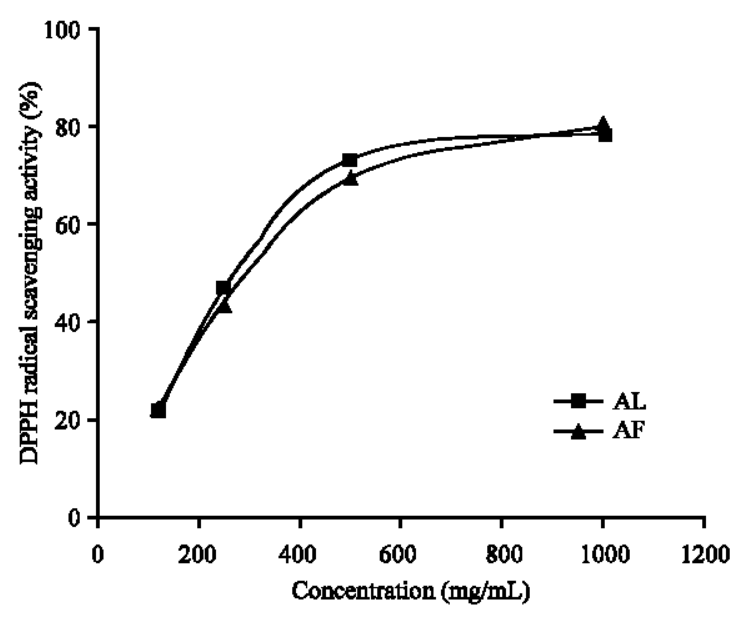

Fig. 1: DPPH radical scavenging activity of $\mathrm{AL}$ and $\mathrm{AF}$ 
respectively, at concentrations of $125,250,500$ and $1000 \mathrm{mg} / \mathrm{m}$. The AF extract showed $21.2 \pm 0.4,46.2 \pm 0.4$, $72.9 \pm 2.1$ and $78.1 \pm 0.4 \%$, respectively, at the same concentrations. Thus, the scavenging ability of the two extracts increased with increasing concentration. The IC50 value for the $\mathrm{AL}$ and $\mathrm{AF}$ extracts was 290.69 and $270.56 \mathrm{mg} / \mathrm{mL}$, respectively, showing a lower IC50 value in the latter. However, there were no significant differences in antioxidant capacity between the two extracts because these extracts exhibited a similar level of antioxidant capacity even at high concentrations. As the free radical scavenging ability of extracts is generally concentration dependent, the IC50 value can be used as a cutoff value when evaluating the antioxidant capacity of extracts.

ABTS assay: The ABTS radical scavenging ability is determined by the extent of decolorization of blue/green color and measurable in most types of extract whether hydrophilic or hydrophobic. The higher phenolic compounds lead to the greater disappearance of color, meaning higher ability to scavenge. The ABTS radical scavenging ability of the $\mathrm{AL}$ extract was $0.7 \pm 0.4,4.7 \pm 0.5$, $32.0 \pm 0.4$ and $93.3 \pm 0.1 \%$, respectively, at concentrations of $1,10,100$ and $1,000 \mu \mathrm{g} / \mathrm{mL}$. The AF extract showed $0.9 \pm 0.1,4.7 \pm 0.5,33.4 \pm 0.4$ and $94.7 \pm 0.2 \%$, respectively. Both sample extracts exhibited a concentration-dependent increase in radical scavenging ability in the ABTS assay. Thus, the antioxidant capacity of the two sample extracts was similar, according to their scavenging ability against $\mathrm{DPPH}$ and ABTS free radicals.

Elastase inhibition activity: Elastin as elastic connective skin tissues, decides the thickness and resilience of the dermis along with collagen. With the ability to stretch $3 \sim 4$ times their length, this elastic protein can form strong elastic structures of cellular tissues. Elastase is an enzyme that degrades not only elastin but also collagen, fibrinogen and other components of immunoglobulin. However, it can also provide regenerating support to the cells damaged or destroyed, making a proper elastase activity important in human body. If elastase activity is unusually increased, elastin fibers in the skin are decomposed as a result of protein breakdown, resulting in decreased skin elasticity (Younes, 1975; Park et al., 2014). Therefore, many studies are conducted to deter the aging process and wrinkle formation through the suppression of excessive elastase activity.

The measured elastase inhibition activity of the AL extract showed $6.5 \pm 0.9,14.4 \pm 0.5,22.2 \pm 0.2$ and $61.2 \pm 0.3 \%$, respectively, at concentrations of $1,10,100$ and 1,000 $\mathrm{mg} / \mathrm{mL}$. The AF extract exhibited $5.9 \pm 0.5,12.5 \pm 0.8,20.6 \pm 0.2$ and $56.8 \pm 0.3 \%$ at the four concentrations. Thus, the

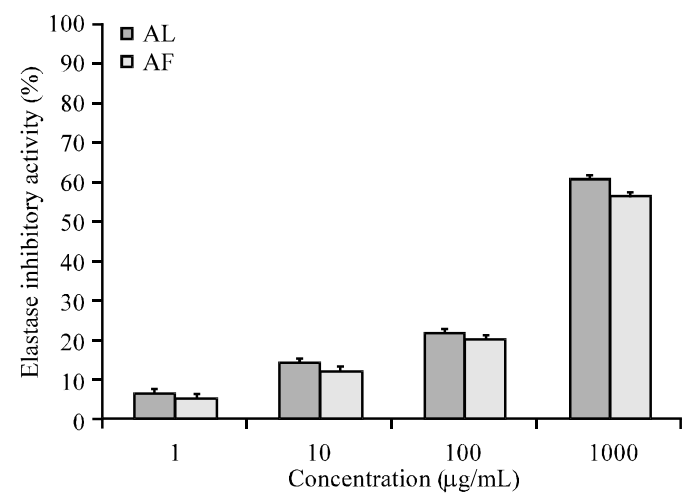

Fig. 2: Elastase inhibitory activity of $\mathrm{AD}$ and $\mathrm{AF} . \mathrm{AD}$ and $\mathrm{AF}$ were incubated at $1,10,100$ and $1,000 \mathrm{mg} / \mathrm{ML}$ with elastase,-succinyl-(L-Ala)3-p-nitroanilide for $30 \mathrm{~min}$. Activities were determined by measurement of absorbance at $445 \mathrm{~nm}$. The results were expressed as mean $\pm \mathrm{SD}$ from three independent experiments

elastase inhibition activities of the two sample extracts increased in a concentration-dependent manner (Fig. 2). These results demonstrated higher overall inhibition activity than those of existing studies in which Kim and Ryoo (2002) used fractions of Theobroma cacao. L fractions and Park and Cha (2016) and Lee et al. (2011) used active ingredients of Rosa multiflora Thunb. In addition, the inhibition of elastase was still higher than those reported by Yuan et al. (2007) and Cha et al. (2005) in their study using extracts from Gleditsiae fructus, Machilus thunbergii and Rhus javanica. Therefore, amaranth has potential as a functional ingredient for anti-aging beauty products. In particular amaranth flower extract demonstrated great potential showing a percent inhibition of $61.2 \pm 0.3 \%$

Collagenase inhibition activity: The measured collagenase inhibition activity of the $\mathrm{AL}$ extract revealed $3.7 \pm 0.9,15.9 \pm 0.6,34.5 \pm 0.3$ and $67.6 \pm 0.3 \%$, respectively, at concentrations of $1,10,100$ and $1,000(\mathrm{mg} / \mathrm{mL})$. The AF extract exhibited $2.1 \pm 0.5,12.7 \pm 1.0,30.1 \pm 0.4$ and $55.8 \pm 0.3 \%$ at these four concentrations. The collagenase inhibition activities of the two sample extracts increased with an increase in concentrations (Fig. 3). These results outperformed inhibition activity reported in existing studies in which Choi (2011) and Park et al. (2002) investigated the functional enzymes of Coriolus versicolor fruit body and Yuan et al. (2007) and Cha et al. (2005) used acanthopanacis cortex and moutan cortex. Both collagenase and elastase are Matrix Metalloproteinase (MMPs) that specifically, act on certain proteins. The expression of these enzymes is induced by oxygen free 


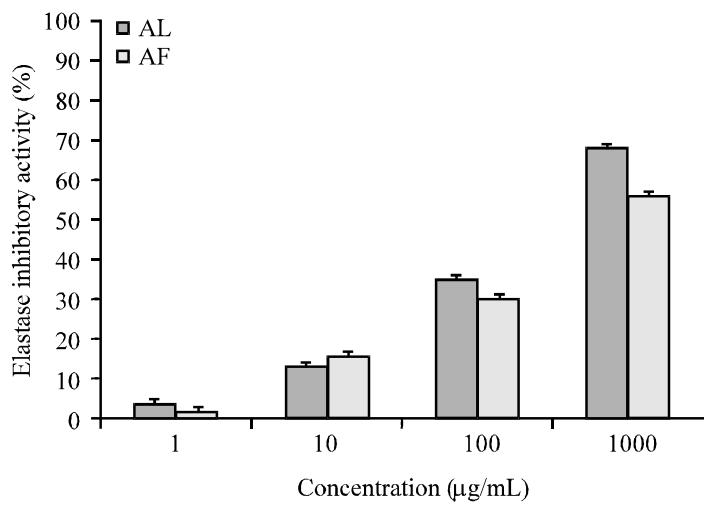

Fig. 3: Collagenase inhibitory activity of $\mathrm{AL}$ and $\mathrm{AF}$. $\mathrm{AL}$ and $\mathrm{AF}$ were incubated at $1,10,100$ and $1,000 \mathrm{mg} / \mathrm{mL}$ with collagenase, 4phenylazobenzyloxycarbonyl-Pro-Leu-Gly-Pro-DArg for $20 \mathrm{~min}$. Activities were determined by measurement of absorbance at $320 \mathrm{~nm}$. The results were expressed as mean $\pm \mathrm{SD}$ from three independent experiments

radicals and UV rays, causing severe protein breakdown or damage which in turn decreases dermis elasticity and induces wrinkle formation and aging (Lee et al., 1999).

In the present study, amaranth extract when compared with other natural plant extracts, exhibited excellent inhibition performance against elastase and collagenase, both of which are mainly used for the evaluation of anti-aging effects. Although, the inhibition activity of the $\mathrm{AL}$ and $\mathrm{AF}$ extracts showed no significant difference, the $\mathrm{AF}$ was more effective in inhibiting elastase and the $\mathrm{AL}$ showed better anti-collagenase activity. As part of the aging process, the ability of skin fibroblasts to produce collagen declines, leading to increasing formation of wrinkles. However, the amount of collagen in the skin is not solely determined by collagen synthesis because the extent of MMPs expression is also an important factor influencing skin aging. Thus, blocking UV rays and oxygen free radicals which increase the release of MMPs is an important method to slow down skin aging.

In the present study, Amaranth extract showed high ability to inhibit protein expression and enzyme activity as well as potent anti-oxidant ability. Thus, this extract is considered a possibility to become a key ingredient for anti-aging beauty products. As the facial mask market has been recently expanding globally, a wide variety of products are available. Some masks are developed using storytelling tactics and this trend combines brands of food products such as yogurt and vegetable beverage to create a foodmetics market. Furthermore, the growing attention of consumers on inner beauty increases the significance of developing food-inspired cosmetic ingredients.

\section{CONCLUSION}

In order to evaluate the potential of amaranth as a functional cosmetic ingredient, this study was conducted to investigate the antioxidant capacity and anti-aging effects of edible amaranth leaves and flowers using two different antioxidant assays and two enzymes, respectively. As a result, the two extracts exhibited IC50 values of 290.69 and $270.56 \mathrm{mg} / \mathrm{mL}$ for antioxidant capacity, showing no significant difference, irrespective of concentration level, although, their capacity was higher than those of natural extracts reported in existing studies. In terms of anti-aging potential, higher activity was observed in the AL extract against elastase while there was no significant difference between the two extracts with respect to collagenase inhibition activity. Given the increasing concern over synthetic products, the development of natural ingredients would be a meaningful approach. In light of this fact, this study has provided valuable information on the biological potency of amaranth extract as a functional cosmetic ingredient. material for antioxidant and anti-aging.

\section{ACKNOWLEDGEMENT}

This research was supported by 2018 Basic Science Research Program through the National Research Foundation of Korea funded by the of Education, Science and Technology (No. : 2018008274)

\section{REFERENCES}

Cha, B.C., E.H. Lee and M.A. Noh, 2005. Antioxidant activity of Smilacis chinae radix. Korean J. Pharmacogn., 36: 195-200.

Choi, H.S., 2011. Effect of adding amaranth powder on noodle quality. Korean J. Food Nutr., 24: 664-669.

Chung, J.H., V.N. Hanft and S. Kang, 2003. Aging and photoaging. J. Am. Acad. Dermatol., 49: 690-697.

Han, J.H., H.K. Moon, S.K. Chung and W.W. Kang, 2013. Comparison of antioxidant activities of radish bud (Raphanus sativus L.) according to extraction solvents and sprouting period. J. Korean Soc. Food Sci. Nutr., 42: 1767-1775.

Kim, J.S. and H.J. Ryoo, 2002. Application to the biscuits manufacture of processed amaranth seeds. Korean Soc. Food Nutr., 15: 321-325. 
Kwon, H.N., 2013. The effect of the antioxidant activities of fermented mulberry extracts as cosmetic materials. J. Invest. Cosmetol., 9: 221-227.

Lee, E.S., E.M. Ju and J.H. Kim, 2001. Free radical scavenging and antioxidant enzyme fortifying activities of extracts from Smilax china root. Exp. Mol. Med., 33: 263-268.

Lee, K.K., J.H. Kim, J.J. Cho and J.D. Choi, 1999. Inhibitory effects of 150 plant extracts on elastase activity and their anti-inflammatory effects. Int. J. Cosmet. Sci., 21: 71-82.

Lee, Y.S., B.O. Kim and N.W. Kim, 2014. Anti-wrinkle and antioxidant activity of the extract of Albizzia julibrissin leaves. J. Invest. Cosmetol., 10: 317-326.

Omaye, S.T., K.A. Reddy and C.E. Cross, 1977. Effect of butylated hydroxytoluene and other antioxidants on mouse lung metabolism. J. Toxicol. Environ. Health Part A. Curr. Issues, 3: 829-836.

Park, J.A., K.S. Jin, H.J. Kwon and B.W. Kim, 2014. The anti-obesity effect of smilax China extract. Korean J. Microbiol. Biotechnol., 42: 354-360.

Park, J.H., J.M. Kim and W.I. Do, 2002. Pharmacognostical study on the to bog Ryung. Korean J. Pharmacogn., 33: $169-172$.
Park, S.H. and K.O. Cha, 2016. Oriental and Western food effects analysis of misutgaru for fusion remedy in diabetes mellitus. J. Korea Convergence Soc., 7: 137-143.

Starkey, P.M., A.J. Barrett and M.C. Burleigh, 1977. The degradation of articular collagen by neutrophil proteinases. Biochim. Biophys. Acta BBA. Enzymol., 483: 386-397.

Valko, M., D. Leibfritz, J. Moncol, M.T.D. Cronin, M. Mazur and J. Telser, 2007. Free radicals and antioxidants in normal physiological functions and human disease. Int. J. Biochem. Cell Biol., 39: 4484.

Younes, M.E.G., 1975. Chemical examination of local plants XIV: Triterpenoids from the leaves of Egyptian Callistemon lanceolatus. Aust. J. Chem., 28: 221-224.

Yuan, L.L., G.P. Gan, H.Z. Wu, H.Z. Zhang and C.L. Li et al., 2007. A flavonoid glycoside isolated from Smilax china L. rhizome in vitro anticancer effects on human cancer cell lines. J. Ethnopharmacol., 113: 115-124. 